\title{
Notes on black elytron species of Pyrrhalta Joannis and the description of a new species from China (Coleoptera, Chrysomelidae, Galerucinae)
}

\author{
Rui-E Nie ${ }^{1, \dagger}$, Da-Kang Zhou ${ }^{1,2, \ddagger}$, Huai-Jun Xue ${ }^{1, \S}$, Xing-Ke Yang ${ }^{1,1}$ \\ I Key Laboratory of Zoological Systematics and Evolution, Institute of Zoology, Chinese Academy of Sciences, \\ Beijing, 100101, China 2 Beijing Botanical Garden, Beijing, 100093, China \\ † urn:lsid:zoobank.org:author:AD7847BD-8394-4EBA-AB07-A947661C0894 \\ † urn:lsid:zoobank.org:author:6130E443-86C4-4DFA-8721-7E8D7AECDB11 \\ § urn:lsid:zoobank.org:author:3401AC1C-253B-4D40-A399-02274728F5F8 \\ | urn:lsid:zoobank.org:author:1DFA8B54-148D-4346-82B1-35DDBBFA9644 \\ Corresponding author:Xing-Ke Yang (yangxk@ioz.ac.cn)
}

Academic editor: A. Konstantinov | Received 8 November 2012 | Accepted 26 March 2013 | Published 12 April 2013

urn:lsid:zoobank.org:pub:D4DDE242-C25A-4A1D-9E50-8CC044B8E108

Citation: Nie R-E, Zhou D-K, Xue H-J, Yang X-K (2013) Notes on black elytron species of Pyrrhalta Joannis and the description of a new species from China (Coleoptera, Chrysomelidae, Galerucinae). ZooKeys 289: 41-56. doi: 10.3897/ zookeys.289.4266

\begin{abstract}
Thirteen species of Pyrrhalta Joannis, 1865 with black elytron are reviewed. A key to species, photographs of aedeagus and habitus are provided. Pyrrhalta qianana sp. n. is described from Guizhou, China. Pyrrhalta martensi Medvedev et Sprecher-Uebersax, 1999 is newly recorded from China (Tibet).
\end{abstract}

\section{Keywords}

Coleoptera, Galerucinae, Pyrrhalta, black elytron, new species, key

\section{Introduction}

The genus Pyrrhalta Joannis, 1865 is a large, worldwide genus distributed in the Holarctic, Oriental and Australian Regions. The genus was firstly proposed as a subgenus of Galeruca Geoffroy, 1762 by Joannis (1865), and Galeruca viburni Paykull, 1799 was deemed to be fixed as type species by monotypy. The genus presents serious difficulties in several aspects of its classification and nomenclature. Firstly, this genus is very large

Copyright Rui-E Nie et al. This is an open access article distributed under the terms of the Creative Commons Attribution License 3.0 (CC-BY), which permits unrestricted use, distribution, and reproduction in any medium, provided the original author and source are credited. 
including 115 species in the world (Xue and Yang 2010). Secondly, its status is still not entirely clear being obscured by apparent relationship of Pyrrhalta and Galerucella Crotch, 1873 (Weise 1924, Gressitt and Kimoto 1963, Wilcox 1965). Separation of species of typical Pyrrhalta and Galerucella was based on a character of the pronotum. The whole pronotum of Pyrrhalta is covered by hairs, while at least the middle region of the pronotum of Galerucella is glabrous (Gressitt and Kimoto 1963). However, a use of this character was not consistent in the past by different specialists. Some species whose pronotum covered with hair were treated as Galerucella, while some species having glabrous area of pronotum were placed in Pyrrhalta. Meanwhile, some tentative Galerucella species were included into Pyrrhalta clade based on molecular data (Nie et al. 2012). Whether the pronotum character can be regarded as the unique character to distinguish the two genera need be further studied, and the morphological characters for distinguishing Galerucella and Pyrrhalta need to be addressed in the future. Thirdly, the subgenera of Pyrrhalta are still not well defined. For example, Clitenososia Laboissière, 1931, Xanthogaleruca Laboissière, 1934 and Tricholochmaea Laboissière, 1932 were considered as synonyms of Pyrrhalta (Gressitt and Kimoto 1963), while in another study, the groups Galerucella, Neogalerucella Chûjô, 1962, Xanthogaleruca and Tricholochmaea were treated as subgenera of Pyrrhalta (Wilcox 1965). Galerucella, Xanthogaleruca, and Tricholochmaea were treated as valid genera and Neogalerucella as subgenus of Galerucella in some recent studies (Silfverberg 1974, Beenen 2008, Gok et al. 2007, Beenen 2010). The most important argument is whether Xanthogaleruca is treated as a valid genus or a subgenus of Pyrrhalta. The character that defines Xanthogaleruca as a genus is the aedeagus with a comb-shaped internal sac (Silfverberg 1974, Beenen 2003, Gök et al. 2007). However, the relative value of this character is not proved yet. In present study, Xanthogaleruca is treated as a synonym of Pyrrhalta until evidence suggesting otherwise are presented.

This study focuses on the Pyrrhalta species with black elytra, which may not be a natural group. We defined the main character state of the group as "elytra entirely or at least $2 / 3$ black". There are 13 species included in this group. Among them, $P$. qianana sp. n., a new species described below. A key to the species of the group is provided.

\section{Material and methods}

Morphological characters were examined with an Olympus SZ 61 microscope. Genitalia of males and / or females of each species were dissected using the following procedure: for dried or ethanol preserved specimens, the abdomen was separated, transferred to a vial containing $10 \% \mathrm{KOH}$ which was heated in a boiling water bath for $10 \mathrm{~min}$. The genitalia were then carefully removed in a cavity slide under distilled water using fine forceps and hooked minuten-pin dissecting needles. Series of partially focused photographs were made with a digital camera (Nikon D300S) attached to a stereomicroscope (Zeiss Discovery V12), and then combined using Helicon Focus software, and finally were evaluated and assembled using Adobe Photoshop CS 8.0 and Illustrator CS4 software. 


\section{Material examined is deposited in the following collections}

BMNH The Natrual History Museum, London, UK

IZAS Institute of Zoology, Chinese Academy of Sciences, Beijing, China

JBCB Jan Bezděk collection, Brno, Czech Republic

MHBU Museum of Hebei University, Baoding, Hebei, China

NHMB Naturhistorisches Museum Basel, Switzerland

USNM The United States National Museum of Natural History (Smithsonian Institution), Washington, D.C., USA

ZIN Zoological Institute, Russian Academy of Sciences, Saint-Petersburg, Russia

\section{Taxonomy}

\section{Key to species of Pyrrhalta with entirely or at least $2 / 3$ black elytra}

$1 \quad$ Head and pronotum yellowish brown, with or without black markings ......2

- $\quad$ Head and pronotum dark, with or without brown markings.......................10 10

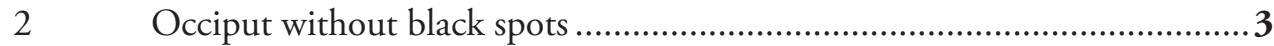

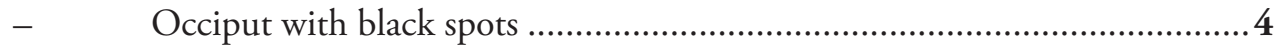

3 Elytron entirely black with flattish surface, space between punctures smaller than diameter of puncture .............................. P. huangshana Chen, 1964

- $\quad$ Elytron black, suture and lateral margins light yellow; elytral surface convex, space between punctures larger than diameter of puncture...P. qianana sp. $\mathbf{n}$. 4 Middle of pronotum with black reversed trapezoid marking P. meghalayana Medvedev, 2002

- $\quad$ Pronotum with three black spots, located in both lateral sides and middle of

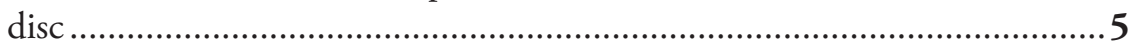

5 Elytron black, a short yellowish brown longitudinal stripe present at base of elytron, which less than $1 / 4$ of elytral length

P. martensi Medvedev et Sprecher, 1999

- $\quad$ Elytron black, without yellowish brown stripe or with a long yellowish brown longitudinal stripe through the whole elytron ..............................................6

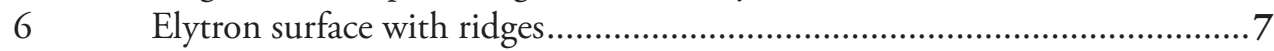

- Elytron surface without ridges .............................................................. 8

$7 \quad$ Elytron dark metallic bronze with slight shade of olive green; with two distinct longitudinal ridges close to suture and flat margin

P. subaenea (Ogloblin, 1936)

- $\quad$ Elytron black, lateral margin and epipleuron brown, disc with 7 irregular black spots formed by groups of dense punctures, short yellowish band present at base of elytron which is the same length as scutellum; longitudinal ridge in middle of disc and under humerus....... P. tianmuensis Chen, 1964 
8 Elytron black, each with yellow longitudinal stripe starting side of humerus and ending before elytral apex, elytral margin and epipleuron brown P. warchalowskii Bezděk, 2007

- $\quad$ Elytron black or reddish brown or brown black, without yellow longitudinal stripe, elytral margin and epipleuron brown or black

9 Antenna $1 / 3$ as long as elytra; basal margin of pronotum slightly concave in middle, lateral margin clearly convex in $1 / 2$; elytron entirely black, disc convex with dense, deep punctures

P. orientalis (Ogloblin, 1936)

- $\quad$ Antenna $1 / 2$ as long as elytra; basal margin of pronotum sinuate, lateral margin narrow basally, obtusely rounded anteriorly; elytron black, basally, along suture, with stripe in middle of disc, lateral margin and epipleuron brown, disc with dense, small, and shallow punctures

P. sulcatipennis (Chen, 1942)

10 Occiput with two brown spots; pronotal disc with six small depressions .......

P. fossata (Chen, 1942)

- Occiput without two brown spots; pronotal disc with three depressions ... 11

11 Elytron reddish brown with broad longitudinal blackish marking covering nearly all interior $2 / 3$ of disc P. tatesuji Kimoto, 2001

Elytron without blackish marking.............................................. 12

12 Antenna slender; pronotum with $\mathrm{V}$ shaped depressions besides of disc, a triangle concave near middle of basal margin; elytron bronzy black with purplish or bronzy tinges metallic lustre, lateral margin not with expanded margin P. metallica Gressitt et Kimoto, 1963

Antenna very stout; pronotum with a wide longitudinal depression in the middle of disc, round depressions besides of disc; elytron brown black, different shades, with bronze metallic lustre, lateral margin expanded with a swollen ridge parallel to margin P. xizangana Chen et Jiang, 1981

\section{Pyrrbalta fossata (Chen, 1942)}

http://species-id.net/wiki/Pyrrhalta_fossata

Figs $1-2,42$

Galerucella fossata Chen, 1942: 19.

Pyrrhalta fossata: Gressitt \& Kimoto, 1963: 439, 449.

Neogalerucella fossata: Beenen, 2010: 449.

Specimens examined. Type material: Holotype: + , re-written (original label is in Chinese): China, Sichuan, Kangding, 25-VIII-1939, collector unknown (IZAS).

Distribution. China (Sichuan).

Notes. Holotype of this species was diagnosed as male in the original description without being dissected (Chen 1942). Actually the holotype is female. Its spermatheca is illustrated here (Fig. 42). The updated catalogue of Galerucinae (Beenen 2010) 


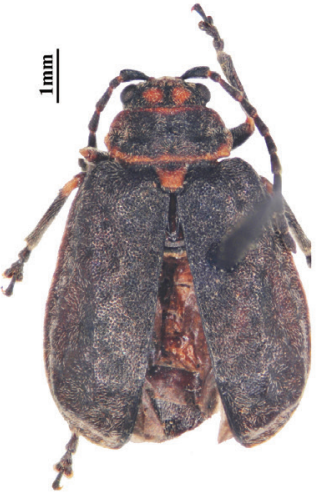

1

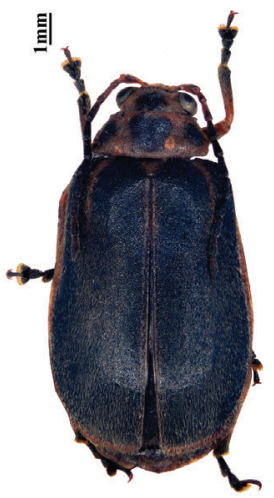

5

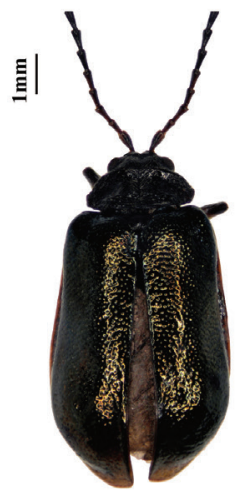

9

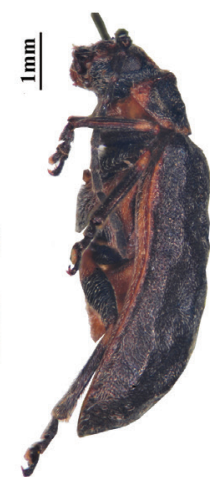

2

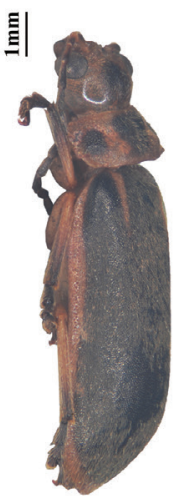

6

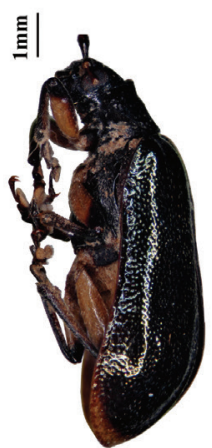

10

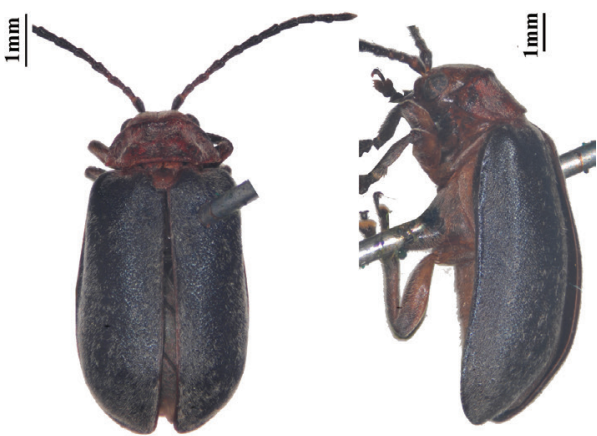

3

4

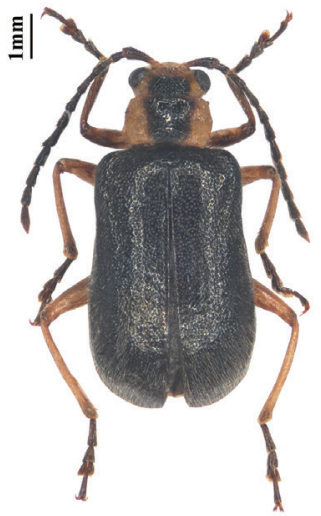

7

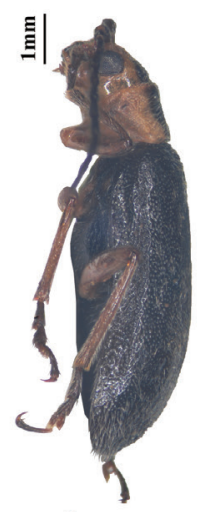

8

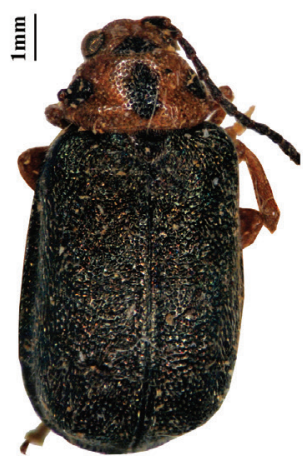

11

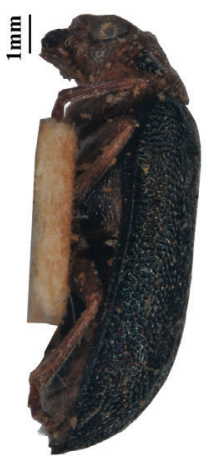

12

Figures I-I 2. Habitus. I-2 P. fossata (holotype) 3-4 P. huangshana (holotype) 5-6 P. martensi (paratype) 7-8 P. meghalayana (paratype) 9-10 P. metallica I I-12 P. orientalis (holotype).

showed that this species belonged to a subgenus Neogalerucella of Galerucella. In this study, we found that the disc of pronotum is entirely covered by hairs. So we think that this species should be remained in Pyrrhalta. 


\section{Pyrrhalta huangshana Chen, 1964}

http://species-id.net/wiki/Pyrrhalta_huangshana

Figs 3-4, 24-25

Pyrrhalta huangshana Chen, 1964: 207.

Specimens examined. Type material: Holotype: $\hat{\partial}$, re-written (original label is in Chinese): China, Anhui, Huang Mountain, 5-VIII-1936, collector unknown (IZAS).

Distribution. China (Anhui).

Notes. Aedeagus of the holotype is illustrated here (Figs 24-25), dorsal view: asymmetrical but nearly parallel-sided, tapered at extreme apex which is subacute; dorsal opening largely on the left side; lateral view: gradually tapering to a subacute tip, slightly arched on the left side, nearly semi - circular on right side.

\section{Pyrrhalta martensi Medvedev et Sprecher, 1999} http://species-id.net/wiki/Pyrrhalta_martensi

Figs 5-6, 26-27

Pyrrhalta martensi Medvedev \& Sprecher, 1999: 366.

Specimens examined. Type material: Paratypes: 1 , original label: "W-Nepal, Kali Gandaki, Knola C.J. Rai / Kopchepani, 1500-1600 m, 21-V-1984 / PARATYPUS, L.N. Medvedev" (NHMB); $10^{\lambda}$, original label: "India, Darjeeling D., Bhakta B. / Kalimpong, 9th mile $1500 \mathrm{~m}, 14$.VII84 / PARATYPUS, L.N. Medvedev"(NHMB).

Other material (2 spec.): 20 , China, Tibet, Linzhi, Muotuo, Yarang, $760 \mathrm{~m}, 19-$ VIII-2006, Ming Bai leg. (IZAS).

Distribution. China (Tibet), India, and Nepal.

Notes. This is the first record of this species in China.

\section{Pyrrhalta meghalayana Medvedev, 2002}

http://species-id.net/wiki/Pyrrhalta_meghalayana

Figs 7-8, 28-29

Pyrrhalta meghalayana Medvedev, 2002: 247.

Specimens examined. Type material: Paratypes: $1 \hat{\gamma}, 1$, original label: "NE INDIA; Meghalayana; 1999, 3Km E Tura; 1150; 2530'N, 90¹4'E; 18. IV.; Dembický \& Pacholátko leg. / PARATYPE” (NHMB). 
Other material (1 spec.): 1 , , NE India, Meghalaya, SW of Sohra, 2513-14'N, 9140', 700-950m, 22-V-2005, C.L. Peša leg. (JBCB).

Distribution. India.

\section{Pyrrhalta metallica Gressitt \& Kimoto, 1963}

http://species-id.net/wiki/Pyrrhalta_metallica

Figs 9-10, 30-31

Pyrrhalta metallica Gressitt \& Kimoto, 1963: 457.

Specimens examined. Type material: Allotype: 1 , , original label: "near O-Er., $\mathrm{Nr}$ Weichow, Aug. 618 '33, 6000-1500ft / SzechwanChina, DCGraham / ALLOTYPE J.L. Gressitt" (USNMNH).

Other material (3 spec.): $2 \hat{\jmath}$, China, Yunnan,Yundi, 3700m, 28-VII-1979, JiangXing Rao leg. (IZAS); $1{ }^{\Uparrow}$, China, Yunnan, Yundi, 3700m, 28-VII-1979, Zhi-Wen Kui leg. (IZAS).

Distribution. China (Sichuan, Yunnan).

\section{Pyrrbalta orientalis (Ogloblin, 1936)}

http://species-id.net/wiki/Pyrrhalta_orientalis

Figs 11-12, 32-33

Galerucella (Xanthogaleruca) orientalis Ogloblin, 1936: 102, 390.

Pyrrhalta orientalis: Gressitt \& Kimoto, 1963: 461.

Specimens examined. Type material: Type: $\hat{\delta}$, original label: "Shan-hai-Kwan, In Mountains, 1.9.06., F.M. Thomson, 1907-200 / Galerucella orientalis sp. n., D. Ogloblin det., 1935. type." (BMNH).

Other material (44 spec.): 10, China, Beijing, Badaling, 700m, 23-VII-1964, Qin Zhou (IZAS); 19, China, Beijing, Badaling, 27-V-1980, collector unknown (IZAS); 2ㅇ, China, Beijing, Shanbao, 26-V-1978, Sheng-Qiao Jiang leg. (IZAS); 10 , China, Beijing, Shanbao, 4-VII-1978, Sheng-Qiao Jiang leg. (IZAS); 10, China, Beijing,

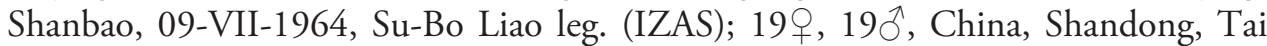
Mountain, 21-IV-1993, Cheng-Gang Zhou leg. (IZAS)

Distribution. China (Liaoning, Hebei, Shanxi, Shandong, Fujian).

Notes. Aedeagus is illustrated here (Figs 32-33), dorsal view: slightly asymmetrical, gradually and slightly widened to apex, apex with a long acute tip; lateral view: nearly straight on left side, gradually tapering to acute tip on right side. 


\section{Pyrrbalta subaenea (Ogloblin, 1936)}

http://species-id.net/wiki/Pyrrhalta_subaenea

Figs 13-14, 34-35

Galerucella (Xanthogaleruca) subaenea Ogloblin, 1936: 102, 389.

Pyrrhalta subaenea: Gressitt \& Kimoto, 1963: 466.

Specimens examined. Type material: Syntypes: 1 , $1 \hat{0}$, original label: "entre Za-mi et Ta-pa, 16-VII-93, Tchouen tshin / Syntypus" (ZIN).

Distribution. China (Sichuan).

Notes. Aedeagus of a syntype is illustrated here (Figs 34-35), dorsal view: slightly asymmetrical, gradually and slightly widened to apex, apex with acute tip in middle; lateral view: gradually tapering to subacute tip, slightly arched on left side, nearly semi-ellipse on right side.

\section{Pyrrbalta sulcatipennis (Chen, 1942)}

http://species-id.net/wiki/Pyrrhalta_sulcatipennis

Figs $15-16,36-37$

Gallerucella sulcatipennis Chen, 1942: 18.

Pyrrhalta sulcatipennis: Gressitt \& Kimoto, 1963: 466.

Specimens examined. Type material: Holotype: $\widehat{O}$, re-written (original labels are in Chinese): China, Sichuan, Emei Mountain, 25-VIII-1939, C.S. TSI leg. (IZAS); Paratype: $1+1 \hat{0}$, the same locality as Holotype (IZAS).

Other material (26 spec.): 1q, China, Hunan, Sangzhi, Tianping Mountain, 1400m, 12-VIII-1988, Shu-Yong Wang leg. (MHBU); 5, 5 ${ }^{\lambda}$, China, Hunan, Sangzhi, Bada Mountain, 10/11-VII-2004, Ji-Liang Wang and Jian-Feng Wang leg. (MHBU); 40, China, Sichuan, Emei Mountain, 25-VIII-1939, You-Cai Lu and Zong-Yuan Wang leg. (IZAS); 2 , , China, Guizhou, Fanjing Mountain, 2300m, 5/4VIII-2001, Hong-Bin Liang leg. (IZAS); 69, China, Guizhou, Fanjing Mountain,

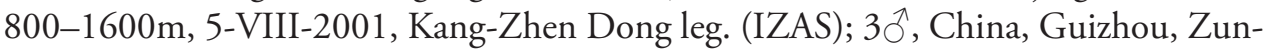
yi, Kuankuoshui Nature Reserve, 1530m, 7-VI-2010, Rui-E Nie leg. (IZAS).

Distribution. China (Hunan, Sichuan, Guizhou).

\section{Pyrrhalta tatesuji Kimoto, 2001}

http://species-id.net/wiki/Pyrrhalta_tatesuji

Fig. 23

Pyrrhalta tatesuji Kimoto, 2001: 45.

Distribution. Nepal. 


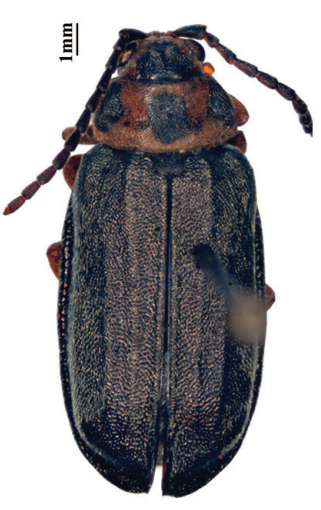

13

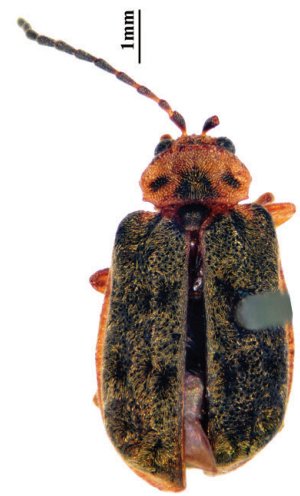

17

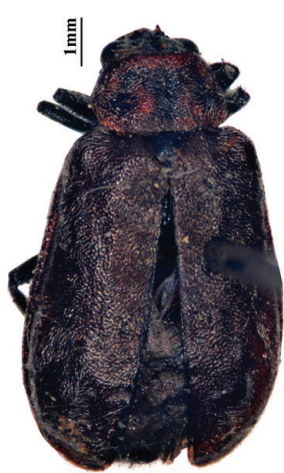

21

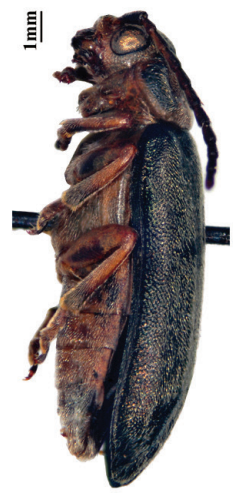

14

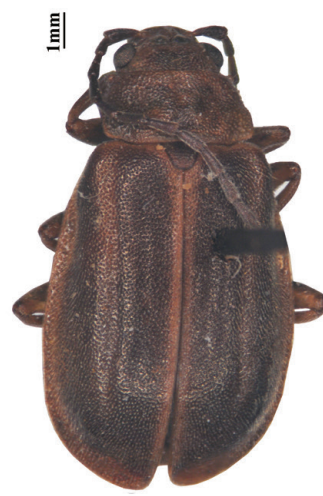

15

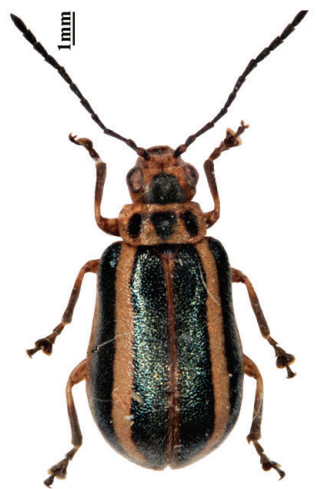

19

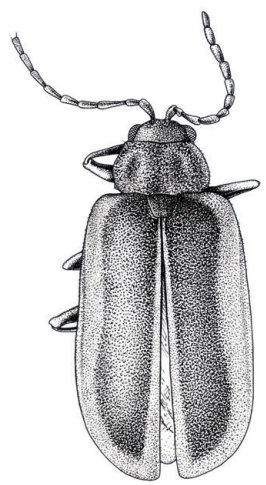

23

Figures 13-23. Habitus. 13-I4 P. subaenea (syntype) I5-16 P. sulcatipennis (holotype) I7-18 $P$. tianmuensis (holotype) 19-20 P. warchalowskii (paratype) 2I-22 P. xizangana (holotype) 23 P. tatesuji Kimoto (drawing after original photograph in Kimoto, 2001).

Notes. We did not examine any specimens of this species. We diagnosed it as belonging to elytron-black group based on the original description and the figure of type (Fig. 23). 
10. Pyrrhalta tianmuensis Chen, 1964

http://species-id.net/wiki/Pyrrhalta_tianmuensis

Figs17-18, 38-39

Pyrrhalta tianmuensis Chen, 1964: 207.

Specimens examined. Type material: Holotype: $\odot$, re-written (original labels are in Chinese): China, Zhejiang, Tianmu Mountain, 6-VIII-1937, collector unknown (IZAS).

Other material (1 spec.): 10, China, Zhejiang, W. Tianmu Mountain, 30-VII1998, Hong Wu leg. (IZAS).

Distribution. China (Zhejiang).

Notes. Aedeagus is illustrated here for the first time. Aedeagus: slender, dorsal view: strongly asymmetrical, apex with acute tip, apical part narrower than basal part, gradually tapering apically but arching near half of base on right side, nearly straight on left side before apex; lateral view: very slender, nearly straight on left side, suddenly tapering to acute tip at basal 1/4 on right side (Figs 38-39).

\section{Pyrrhalta warchalowskii Bezděk, 2007}

http://species-id.net/wiki/Pyrrhalta_warchalowskii

Figs 19-20, 40-41

Pyrrhalta warchalowskii Bezděk, 2007: 607.

Specimens examined. Type material: Paratype: 19 , original label: "S-INDIA, Tamil Nadu state, Nilgiri hills, $10 \mathrm{~km} \mathrm{SW}$ of Manjoor, $76^{\circ} 35^{\prime} \mathrm{E}, 11^{\circ} 12^{\prime} \mathrm{N}$, Thiashola reserved forest / near Carrington estate, ca 2100m, 14-19-VI-1999, Z. Kejval \& M. Trýzna leg. / PARATYPUS” (JBCB).

Distribution. India.

\section{Pyrrhalta xizangana Chen et Jiang, 1981}

http://species-id.net/wiki/Pyrrhalta_xizangana

Figs 21-22, 43

Pyrrhalta xizangana Chen \& Jiang, 1981: 459.

Specimens examined. Type material: Holotype: $\widehat{\partial}$, re-written (original labels are in Chinese): China, Tibet, 52 Daoban, 9-VII-1976, Yin-Heng Han leg. (IZAS). Paratypes: 39 , re-written (original labels are in Chinese): China, Tibet, Chaya, Jitang, 7-VII-1976, Yin-Heng Han leg. (IZAS).

Distribution. China (Tibet). 


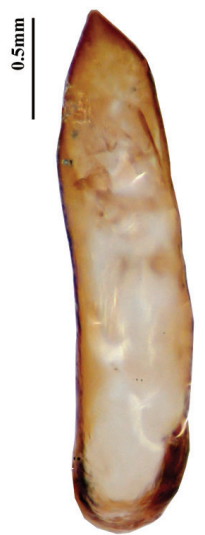

24

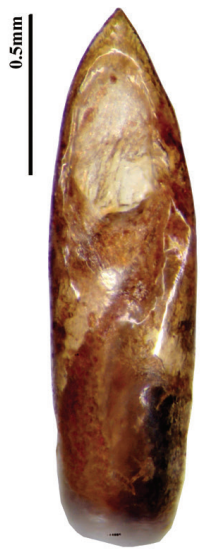

28

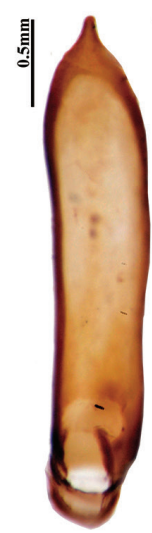

32

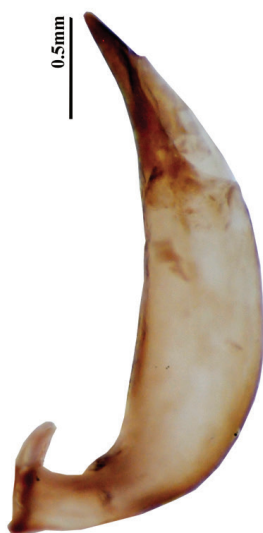

25

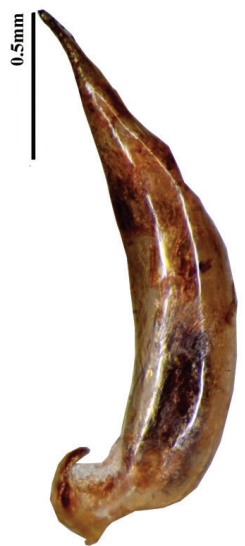

29

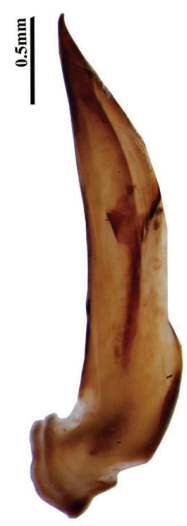

33

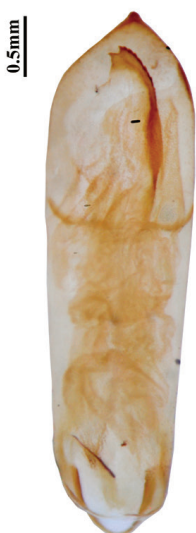

26

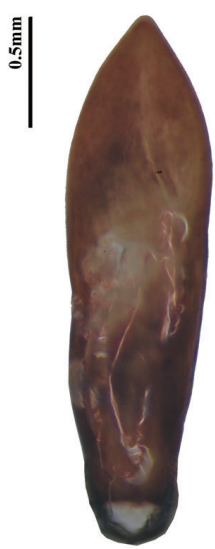

30

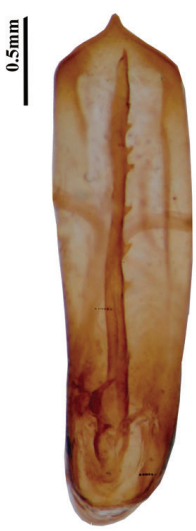

34

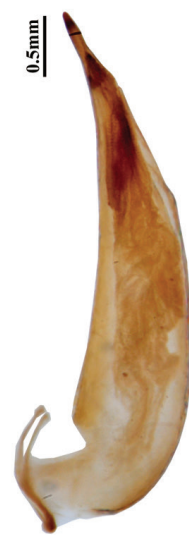

27

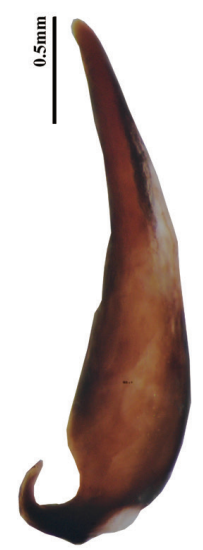

31

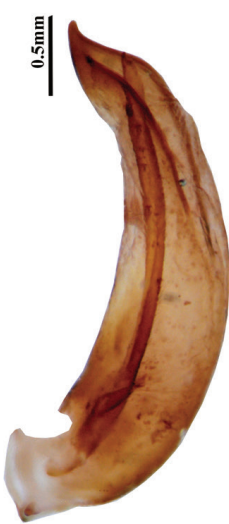

35

Figures 24-35. Aedeagus. 24-25 $P$. huangshana (holotype, 24 dorsal view 25 lateral view) 26-27 $P$. martensi (26 dorsal view 27 lateral view) 28-29 P. meghalayana (paratype, 28 dorsal view 29 lateral view) 30-3 I P. metallica (30 dorsal view $\mathbf{3}$ I lateral view) 32-33 P. orientalis (32 dorsal view $\mathbf{3 3}$ lateral view) 34-35 $P$. subaenea (syntype, $\mathbf{3 4}$ dorsal view $\mathbf{3 5}$ lateral view). 


\section{Pyrrhalta qianana Nie \& Yang, sp. $n$.} urn:lsid:zoobank.org:act:0ABCCB95-FF41-4DE8-9185-E2B29454998C http://species-id.net/wiki/Pyrrhalta_qianana Figs 44-49

Type material. Holotype: $\widehat{O}^{\lambda}$, China, Guizhou, Zunyi, Kuankuoshui Nature Reserve, Baishaogou, 9-VI-2010, Wan-Gang Liu leg. (IZAS). Paratype: $10^{\Uparrow}$, the same data as holotype (IZAS); 1ð , China, Sichuan, Fengdu, Shiping, 610m, 3-VI-1994, Wen-Zhu Li leg. (IZAS); 10, China, Chongqing, Beipei, Tuanjie, 6-V-1999, Hai-Jian Wang and Yin-Fei Zhu leg. (IZAS); 1 , China, Guizhou, Jiangkou, Huixiangping, 2-III-2001, Guo-Dong Ren leg. (MHBU); 1으, China, Sichuan, Emei Mountain, 1800-1900m, 14-VIII-1957, Fu-Xing Zhu leg (IZAS).

Diagnosis. This species can be separated from all known species in the genus by the following characters: very long antennae (length=4.9 mm), antennomere 3 more than 2 times as long as antennomere 2, and last abdominal sternite of male with very deep U-shape cavity (Fig. 46).

Description. Generally black, apex of labrum and mandible, maxilla, dark brown; head, pronotum, elytral margin, elytral suture, yellowish; antenna black except ventral side of antennomeres 1-5; legs brown except ventral sides of tibiae and tarsi black; scutellum yellowish brown, dark brown on basal part. Body densely covered with short pale silvery pubescences.

Head slightly narrower than prothorax; occiput flat; epicranial suture distinct; frontal tubercles distinctly raised, subquadrate, vertex impunctate.

Antennae long, slender, $0.85 \times$ as long as body, length ratio of antennomeres 1 to 11: 15-11-24-23-22-20-20-16-19-17-20.

Pronotum transverse, nearly $2 \times$ as broad as long, maximum width across pronotum $1.55 \mathrm{~mm}$, distance from basal margin to anterior margin $0.75 \mathrm{~mm}$, anterior margin nearly straight and slightly emarginate at middle, lateral margin constricted in anterior third, basal margin slightly concave mesally; anterior angle nearly rectangular, and posterior angles obtusely rounded. Surface densely pubescent, irregularly punctured, with pair of deep depressions laterally and longitudinal depression in middle.

Scutellum trapezoid, densely punctured, sparsely pubescent.

Elytron subparallel, nearly $3.7 \times$ as long as broad, maximum width across both elytra $1.15 \mathrm{~mm}$, linear distance from base to apex of elytra $4.25 \mathrm{~mm}$; surface confusedly punctured and closely covered with fine hairs; space between punctures smaller than diameter of puncture; epipleuron slightly broad basaly, gradually narrowed toward apex.

Ventral surface: mesoventrite glabrous, mesepisternum and mesepimeron thinly covered with short pubescence. Middle disc of metaventrite brown, with sparse hairs. Last sternite of male with very deep " $U$ " shape emarginate cavity reaching nearly its basal margin.

Legs moderately stout, hind tarsomere 1 nearly equal with last which is nearly as long as 2 and 3 together. 


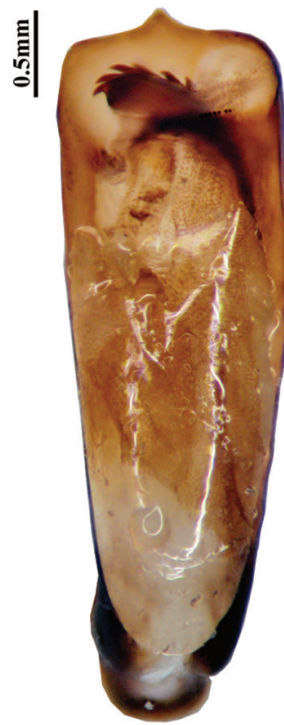

36

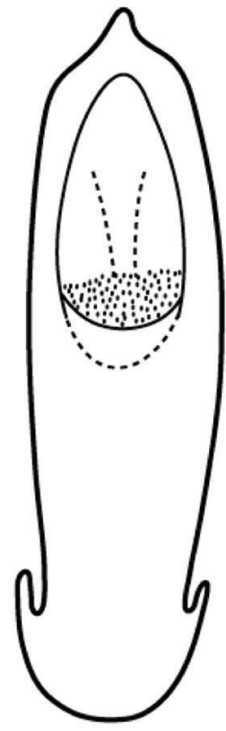

40

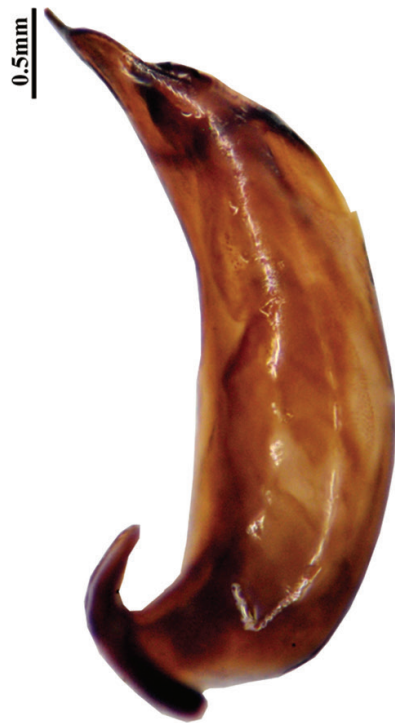

37

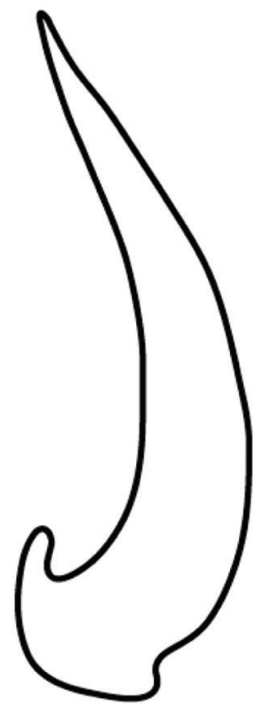

41

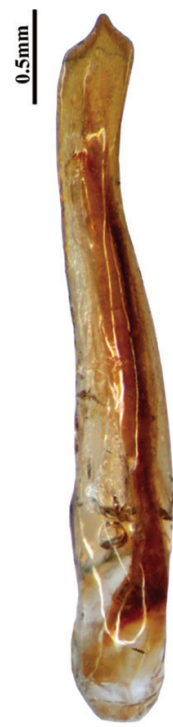

38

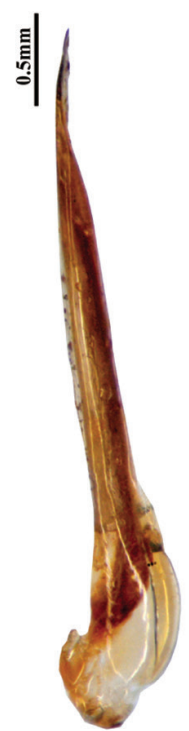

39

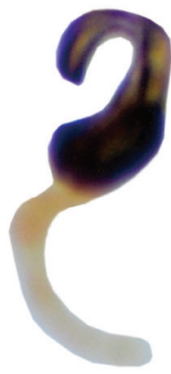

42

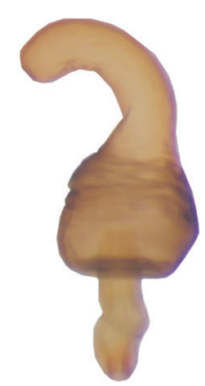

43

Figures 36-43. Aedeagus / spermatheca 36-37 P. sulcatipennis (holotype, $\mathbf{3 6}$ dorsal view $\mathbf{3 7}$ lateral view) 38-39 P. tianmuensis (38 dorsal view 39 lateral view) 40-4I P. warchalowskii (Bezděk 2007 orig., 40 dorsal view 4 I lateral view) $\mathbf{4 2}$ spermatheca of $P$. fossata (holotype) 43 spermatheca of $P$. xizangana (paratype).

Male. Last abdominal sternite with very deep U-shape cavity (Fig. 46). Aedeagus: dorsal view: strongly asymmetrical but nearly parallel-sided, apex subacute, tapered; lateral view: somewhat sinuate on left side, gradually tapering to acute tip on right side. (Figs 48-49). 


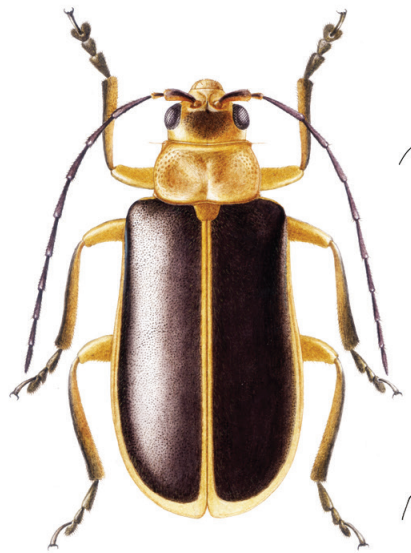

44

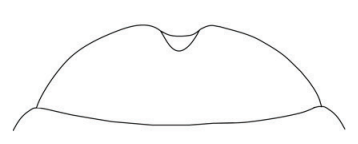

45

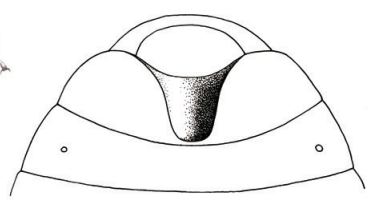

46

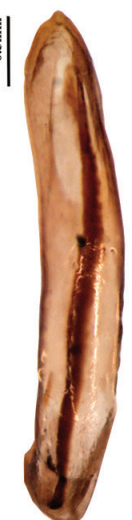

48

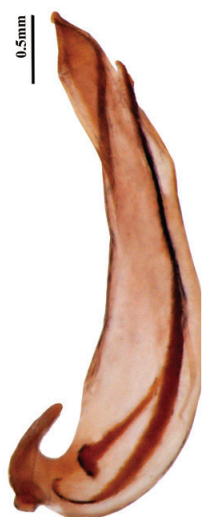

49

Figures 44-49. Pyrrhalta quinana sp. n. 44 Adult 45 The last abdominal sternite of female 46 The last sternite of male $\mathbf{4 7}$ spermatheca $\mathbf{4 8 - 4 9}$ aedeagus (48 dorsal view $\mathbf{4 9}$ lateral view).

Female. Last abdominal sternite with triangle emargination at center of apex (Fig. 45). Spermatheca: base not bent, capsule wall thick, apex of capsule about $1 / 2$ as long as capsule (Fig. 47).

Length: 5.4-5.5 mm (linear distance from labrum to elytral apex); width: 2.0-2.1 $\mathrm{mm}$ (width across base of elytra).

Etymology. This species is named for its holotype locality, Guizhou province (shortened form as "Qian" in Chinese).

Distribution. China (Guizhou, Sichuan).

\section{Discussion}

Pyrrhalta is a species rich, worldwide distributed genus with complex classification and nomenclature. The relationships of Pyrrhalta and related genera (Galerucella, Neogalerucella, Xanthogaleruca, and Tricholochmaea) are still unclear. The updated catalogue of Galerucinae treated Galerucella, Xanthogaleruca, and Tricholochmaea were as valid genera and Neogalerucella as subgenus of Galerucella (Beenen 2010). To explore true relationship of these groups, a thorough revision of Pyrrhalta is necessary. Considering that subgenera currently are very poorly defined, we will separate the genus into several artificial groups and produce a series of revisionary works. In this study, we have reviewed Pyrrhalta species with black elytra. Based on the current morphological work with thirteen species of Pyrrhalta with black elytra two types of internal sac were found. One is with comb-shaped sclerites and another one lacking the sclerites. Comb-shaped sclerites presents in P. sulcatipennis and P. subaenea. They are absent in the rest of the studied species including $P$. orientalis which is placed in Xanthogaleruca in the updated catalogue of Galerucinae (Beenen 2010). Therefore, comb-shaped internal 
sac cannot be used to distinguish Xanthogaleruca and Pyrrhalta. The proper status of Xanthogaleruca could not resolve until a thorough revision of Pyrrhalta is done. We still need to find reliable characters to identify above related groups. It may be necessary to combine traditional morphological methods with molecular and biological methods to achieve this goal.

\section{Acknowledgments}

We thank Mr Wen-Zhu Li for drawing the figures. We are grateful to Dr Jan Bezděk, Dr Si-Qin Ge, Dr Ming Bai and anonymous reviewers for valuable comments. We are most grateful to all of the curators whose material has been used for the present study, particularly to Dr Maxwell VL Barclay (BMNH), Dr Jan Bezděk (Brno, Czech Republic), Dr Michel Brancucci \& Dr Eva Sprecher-Uebersax (NHMB), Dr Boris A. Korotyaev (ZIN), Dr Guo-Gong Ren \& Dr Yu-Xia Yang (MHBU). This research was supported by a Key Innovation-Project of CAS (No. KSCX2-YW-Z-0911), and the National Natural Science Foundation of China (No. 3010300101, 30970393, J1210002).

\section{References}

Beenen R (2003) New records of Xanthogaleruca subcoerulescens (Weise) in southern Turkey (Coleoptera, Chrysomelidae, Galerucinae). Entomologische Blätter 99: 99-103.

Beenen R (2008) Taxonomical and nomenclatural changes in Palaearctic Galerucinae and description of a new species (Chrysomelidae). Entomologische Blätter 103/104: 63-80.

Beenen R (2010) Galerucinae. In: Löbl I \& Smetana A (Ed.) Catalogue of the Palaearctic Coleoptera 6: 74-75, 443-491. Apollo Books, Stenstrup.

Bezděk J (2007) Notes on the Galerucini from India and Sri Lanka, with description of Pyrrhalta warchalowskii n. sp from Tamil Nadu state, India (Coleoptera: Chrysomelidae: Galerucinae). Genus 18: 603-612.

Chen SH (1942) Galerucinae nouveaux de la Faune Chinoise. Notes d'Entomologie Chinoise 9: $1-67$.

Chen SH (1964) New genera and species of Galerucinae from China. Acta Entomologica Sinica 13: 201-211.

Chen SH, Jiang SQ (1981) Coleoptera: Chrysomelidae, Galerucinae. In: Huang FS (Ed.), Insect of Xizang, Vol. 1. Science Press, Beijing, 495-460.

Gök A, Aslan EG, Aslan B (2007) Xanthogaleruca subcoerulescens (Weise, 1884) (Coleoptera: Chrysomelidae), a little-known galerucine from Turkey, with a description of the female, additions to the description of the male, and ecological remarks. Entomological News 118: 259-262. doi: 10.3157/0013-872X(2007)118[259:XSWCCA]2.0.CO;2

Gressitt JL, Kimoto S (1963) The Chrysomelidae (Coleoptera) of China and Korea. Part II. Pacific Insects Monograph, 1B,, 301-1026 pp. 
Kimoto S (2001) The Chrysomelidae (Insecta: Coleoptera) collected by the Kyushu University Scientific Expedition to the Nepal Himalaya in 1971 and 1972. Bulletin of the Kitakyushu Museum of Natural History 20: 17-80.

Medvedev LN (2002) New and poorly known Chrysomelidae (Coleoptera) from northern India. Entomologica Basiliensia 24: 245-253.

Medvedev LN, Sprecher-Uebersax E (1999) Katalog der Chrysomelidae von Nepal. Entomologica Basiliensia 21: 261-353.

Nie RE, Xue HJ, Hua Y, Yang XK, Vogler AP (2012) Distinct species or colour polymorphism? Life history, morphology and sequence data separate two Pyrrhalta elm beetles (Coleoptera: Chrysomelidae). Systematics and Biodiversity 10: 133-146. doi: $10.1080 / 14772000.2012 .687783$

Ogloblin DA (1936) Insectes Coleopteres Vol. 26. Chrysomelidae, Galerucinae. In: Sernov SA, Stackelberg AA (Eds) Faune de L'URSS. l'Academie des Sciences de l'URSS, Moscou, $1-455 \mathrm{pp}$.

Silfverberg H (1974) The west Palaearctic species of Galerucella Crotch and related genera (Coleoptera, Chrysomelidae). Notulae Entomologicae 54: 1-11.

Weise J (1924) Chrysomelidae: Galerucinae, In Schenkling S (Ed.) Coleopterorum Catalogus, Pars 78. Berlin: Dr. W. Junk, 182 pp.

Wilcox JA (1965) A synopsis of the North American Galerucinae (Coleoptera: Chrysomelidae), Bulletin of the New York State Museum and Science Service 400: 1-68 pp.

Xue HJ, Yang XK (2010) Species catalogue of Pyrrhalta Joannis (Coleoptera: Chrysomelidae: Galerucinae) of the World. Entomotaxonomia 32 (Suppl.): 119-136. 